\title{
The Aquilegia genome reveals a hybrid origin of core eudicots
}

\author{
Gökçe Aköz $z^{1,2}$ and Magnus Nordborg ${ }^{1 *}$ (D)
}

\begin{abstract}
Background: Whole-genome duplications (WGDs) have dominated the evolutionary history of plants. One consequence of WGD is a dramatic restructuring of the genome as it undergoes diploidization, a process under which deletions and rearrangements of various sizes scramble the genetic material, leading to a repacking of the genome and eventual return to diploidy. Here, we investigate the history of WGD in the columbine genus Aquilegia, a basal eudicot, and use it to illuminate the origins of the core eudicots.

Results: Within-genome synteny confirms that columbines are ancient tetraploids, and comparison with the grape genome reveals that this tetraploidy appears to be shared with the core eudicots. Thus, the ancient gamma hexaploidy found in all core eudicots must have involved a two-step process: first, tetraploidy in the ancestry of all eudicots, then hexaploidy in the ancestry of core eudicots. Furthermore, the precise pattern of synteny sharing suggests that the latter involved allopolyploidization and that core eudicots thus have a hybrid origin.

Conclusions: Novel analyses of synteny sharing together with the well-preserved structure of the columbine genome reveal that the gamma hexaploidy at the root of core eudicots is likely a result of hybridization between a tetraploid and a diploid species.
\end{abstract}

\section{Background}

Whole-genome duplication (WGD) is common in the evolutionary history of plants (reviewed in [1, 2]). All flowering plants are descended from a polyploid ancestor, which in turn shows evidence of an even older WGD shared by all seed plants [3]. These repeated cycles of polyploidy dramatically restructure plant genomes. Presumably driven by the "diploidization" process, whereby genomes are returned to an effectively diploid state, chromosomes are scrambled via fusions and fissions, lose both repetitive and genic sequences, or are lost entirely [4-11]. Intriguingly, gene loss after WGD is non-random: there is a bias not only against the retention of certain genes $[12,13]$ but also against the retention of one of the WGD-derived paralog chromosomes $[6,9,14-16]$.

We investigated the history of WGDs in the columbine genus Aquilegia for two reasons. The first is related to its phylogenetic position: columbines have been referred to as "basal" eudicots because they

\footnotetext{
* Correspondence: magnus.nordborg@gmi.oeaw.ac.at

${ }^{1}$ Gregor Mendel Institute, Austrian Academy of Sciences, Vienna Biocenter,

Vienna, Austria

Full list of author information is available at the end of the article
}

appear to be an outgroup to most other "core" eudicot lineages $[17,18]$. This matters because our understanding of eudicot karyotype evolution is limited to the heavily sampled core eudicots. Using the recently published Aquilegia coerulea genome [19], we were able to address key questions about the history of polyploidization in all eudicots. Second, we traced the origins of the columbine chromosomes with a particular focus on the strange chromosome 4, which differs from the rest of the genome in many ways. In particular, it harbors more genetic polymorphism and transposable elements, has lower gene density and reduced gene expression, and appears to migrate more, including between species. It also carries the rDNA clusters, and there is reason to believe that knowing the history of the chromosome could help explain its aberrant behavior [19].

\section{Results \\ 2.1.Within-genome synteny confirms columbine paleotetraploidy}

Ancient WGDs have commonly been inferred from the distribution of divergences between gene duplicates. The 
simultaneous generation of gene duplicates via WGD is expected to produce a peak in the age distribution relative to the background age distribution of single-gene duplicates [20-22]. Such a spike of ancient gene birth was the first evidence of paleotetraploidy in columbines [23] and was later supported by gene count-based modeling [24].

Given an assembled genome, a more direct method to infer ancient polyploidy is to look for regions with conserved gene order $[25,26]$. Such conservation (a.k.a. synteny) decreases over time due to gene loss and rearrangements, but will still be detectable unless the extent of change is too extensive. We detected a total of 121 synteny block pairs harboring at least five paralogous gene pairs within the columbine genome. The distribution of these blocks across the seven columbine chromosomes indicates pairwise synteny between large genomic regions (Fig. 1). This 1:1 relationship suggests a single round of WGD in columbines and is further supported by similar levels of divergence between synteny pairs (Additional file 1: Figure S1 and S2).

\subsection{Columbines share ancient tetraploidy with core eudicots}

All sequenced core eudicots appear to share a triplicate genome structure due to paleohexaploidy postdating the separation of monocots and eudicots $([9,28-32]$ and Supplementary Note 5 in [33]). The tetraploidy in columbines, a basal eudicot, might be independent of this ancient "gamma" hexaploidy (Scenarios 1 and 2 in Fig. 2) or might be a remnant of a WGD at the base of all eudicots, which formed the first step of the gamma hexaploidy in core eudicots (Scenario 3 in Fig. 2).

We used the grape (Vitis vinifera) genome as a representative of the core eudicots to distinguish between the three scenarios in Fig. 2. Grape has experienced a relatively small number of chromosomal rearrangements post-gamma and thus strongly resembles the ancestral pre-hexaploid genome [34]. Given the ploidy level of columbine under each scenario, we can predict the ratio of haploid chromosome sets in grape to that in a columbine. If tetraploidy in columbines is lineage-specific and superimposed on the gamma hexaploidy (Scenario 1), we would expect to find a 3:6 ratio of grape and columbine synteny blocks. Instead, we observe a 3:2 relationship (Fig. 3 and Additional file 1: Figure S3) as expected under Scenarios 2 or 3. A similar 3:2 pattern is found in comparisons between grape and sacred lotus [35]. This strongly suggests that basal eudicots do not share the triplicate genome structure of core eudicots, ruling out Scenario 1.

Distinguishing between the two remaining scenarios is more difficult. We began by comparing the divergence at synonymous sites (Ks) between columbine paralogs, grape paralogs, and columbinegrape homologs. In agreement with the analysis of

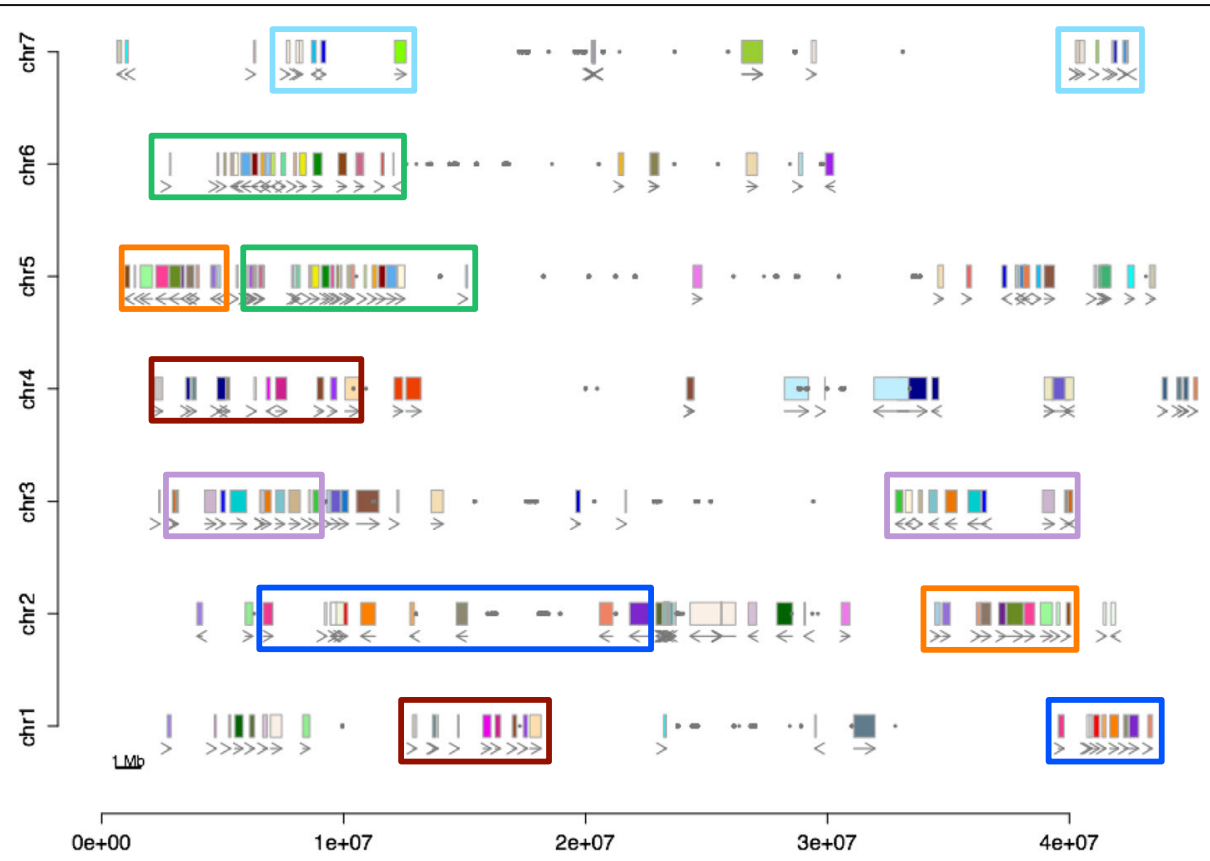

Fig. 1 Intragenomic synteny blocks in the columbine genome. Pairs of synteny blocks are denoted as uniquely colored small rectangles. Larger rectangles of the same color outline large regions of synteny. Arrows under the synteny blocks show the orientation of the alignment between collinear genes. Gray dots highlight BLAST hits of a 329 bp centromeric repeat monomer [19, 27] 


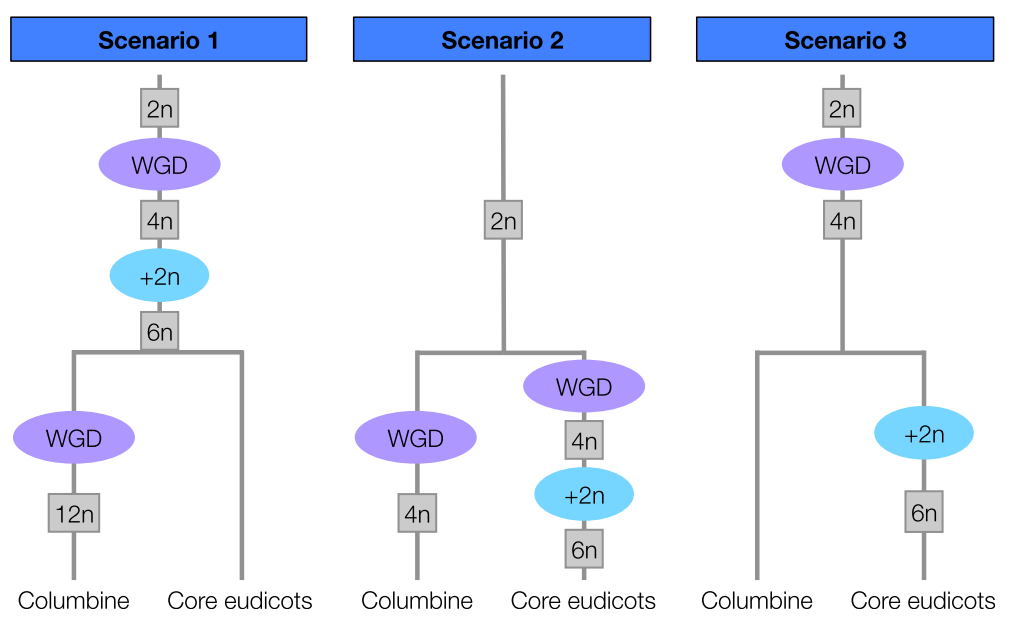

Fig. 2 Three scenarios for the relationship between columbine tetraploidy and core eudicot "gamma" hexaploidy. The gamma hexaploidy is a two-step process: a single round of WGD creates tetraploids (4n) whose unreduced gametes then fuse with diploid gametes $(+2 n)$. Scenario 1 : gamma hexaploidy precedes the split between columbine and core eudicots, with the former undergoing an additional tetraploidy. Scenario 2 : both gamma hexaploidy and columbine tetraploidy occur after the split between columbines and core eudicots. Scenario 3: columbine tetraploidy is derived from the ancient tetraploidy which was the first step of the process leading to gamma hexaploidy

Jiao et al. [36], the Ks distribution for grape paralogs appears to two major peaks, as expected under the two-step model for gamma hexaploidy (Fig. 4). However, columbine paralogs and columbine-grape homologs each show a single peak of divergence-and the peaks overlap each other and the "older" divergence peak of grape paralogs. This suggests that

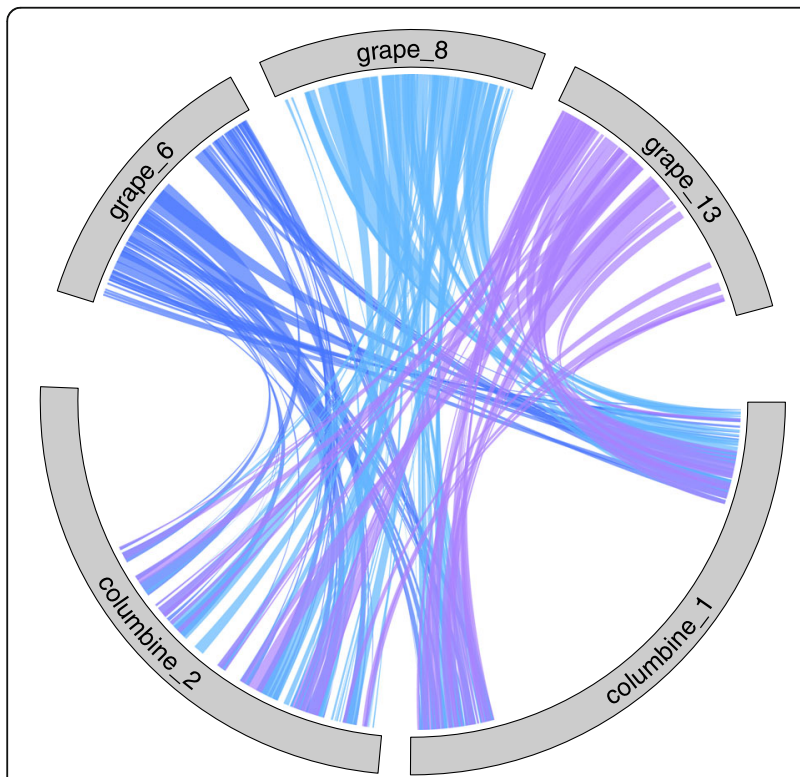

Fig. 3 Synteny between the homologous regions of columbine and grape. The results for columbine chromosomes 1 and 2 and grape chromosomes 6, 8, and 13, shown here, reflect the overall synteny relationship of 3:2 between grape:columbine chromosomes (see Additional file 1: Figure S3 and Additional file 3: Table S2). This pattern argues against Scenario 1 but is consistent with either Scenario 2 or Scenario 3 in Fig. 2 columbine tetraploidy is derived from the tetraploidy that eventually led to gamma hexaploidy in core eudicots (Scenario 3).

Next we considered the gene order similarity between pairs of columbine and grape chromosomes. If columbine and grape have descended from a common tetraploid ancestor (Scenario 3), they should have inherited all diploidization-driven changes to gene order between the paralogous chromosomes of the tetraploid ancestor. As a result, we expect to see alternative paralogous gene orders to be uniquely shared between two pairs of columbine and grape chromosomes (Fig. 5) - whereas if tetraploidization occurred twice (Scenario 2), no such pattern is expected.

We used two different approaches to detect this pattern. First, we clustered homologous segments based on gene order similarity ("Materials and methods"). The pairwise comparisons show that each member of columbine paralogs matches a different grape chromosome (Additional file 1: Figure S6-S8). Reshuffling genes on grape chromosomes further indicates that this pattern of clustering is highly unlikely to be produced by chance $(p=0-0.05)$. Second, we attempted to corroborate the clustering based on gene order similarity by clustering homologous regions based on similarity in protein sequence ("Materials and methods"). Because of the deep history of shared tetraploidy, only a small fraction of all the informative gene trees $(0.016-0.044)$ show the "expected" pairings (Additional file 1: Figure S6-S8), and it is thus not possible to infer history from individual trees. Although it is possible that more sophisticated treebuilding methods would perform better, the order of the homologous genes that do show the expected pairwise 


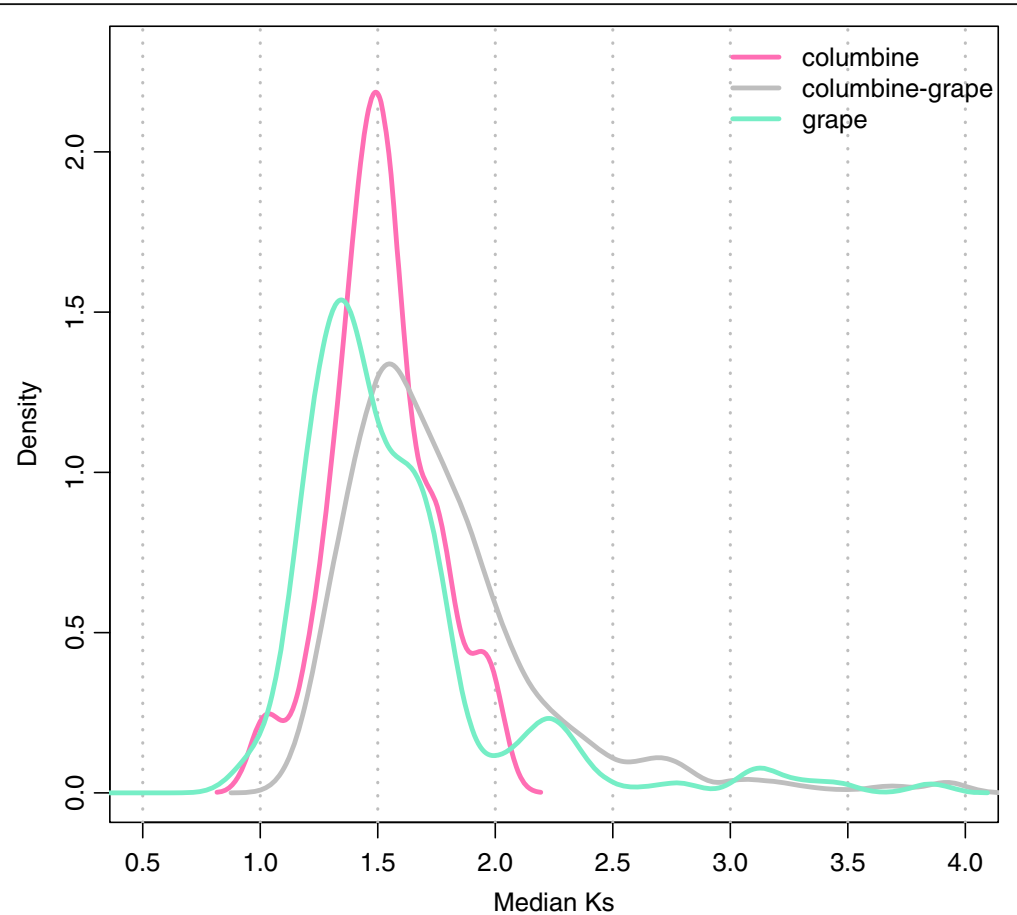

Fig. 4 The distribution of the median Ks across syntenic regions. Synteny blocks are identified within columbine, between columbine and grape, and within grape. Note that only the putative WGD-derived blocks (median Ks=1-2) are kept in columbine (Additional file 1: Figure S2)

\section{$12345 \quad$ Common ancestor (2n)}

\section{$\begin{array}{lllllllllll}1 & 2 & 3 & 4 & 5 & 1 & 2 & 3 & 4 & 5 & \text { Common ancestor }(4 n)\end{array}$}

\section{$1-3-5 \quad 21-4-$ Diploidization}

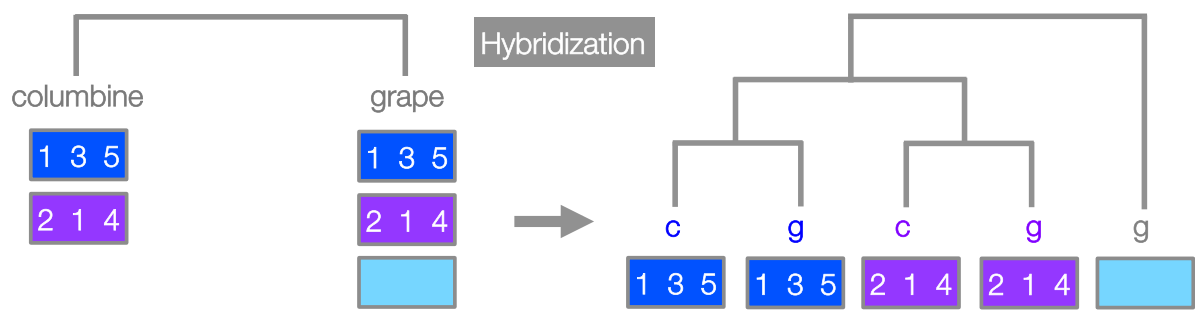

Fig. 5 Gene order-based clustering expected under ancient tetraploidy common to all eudicots. Represented here by blue and purple rectangles, each member of the paralogous chromosome pair experiences intra-chromosomal rearrangements as a part of the diploidization process. Deletions (denoted as "-") will create the gene order "1, 3, 5" on the blue chromosome while both deletions and an inversion will create the gene order " $2,1,4$ " on the purple chromosome. These differential paralogous gene orders will be inherited by both columbine and grape. If we compare the gene order on the homologous chromosomes of columbine and grape at this particular region, we should see "blue" chromosomes of columbine and grape forming one cluster while "purple" chromosomes of columbine and grape forming another cluster. Note that we show here only the "allohexaploidy" model, which predicts that the third grape paralog added via hybridization is an outgroup in this clustering analysis. See Additional file 1: Figure S11 for the expected gene orders under the "autohexaploidy" model 
clustering (based on sequence divergence) again recaptures the clustering pattern inferred from synteny alone (compare Additional file 1: Figure S7 and S9). Thus, the clustering pattern inferred from synteny is mirrored in clustering based on sequence divergence.

An eudicot-wide WGD is further supported by the observation that a chromosomal fusion, presumably experienced by the common tetraploid ancestor, is still detectable in the genomes of columbine and grape despite their separation of around 125 million years [37]. The first hint comes from the composition of the chromosomes: columbine chromosome 5 and grape chromosome 7 both appear to be fusions of the same ancestral chromosomes (Fig. 6). If these chromosomes were created by a single fusion event in the common tetraploid ancestor of eudicots, they should match each other with respect to gene order on each of the two homologous portions ("orange" and "green" portions in Fig. 7). This is what we see: columbine chromosome 5 and grape chromosome 7 cluster together with respect to their gene order on the "orange" portion (Additional file 1: Figure S7). For the "green" portion, columbine chromosome 5 matches grape chromosome 4 (Additional file 1: Figure S8), which used to be fused to grape chromosome 7 [38]. The shared ancestral fusion can also be seen using the cacao (Theobroma cacao) genome [39]. The first chromosome of cacao does not only show a similar pattern of chromosomal ancestry [38, 39] but also shares the gene order exclusively with the grape chromosomes 4 and 7 on the corresponding homologous portions (Additional file 1: Figure S10). In summary, the columbine fusion clusters with that of grape, which, in turn,

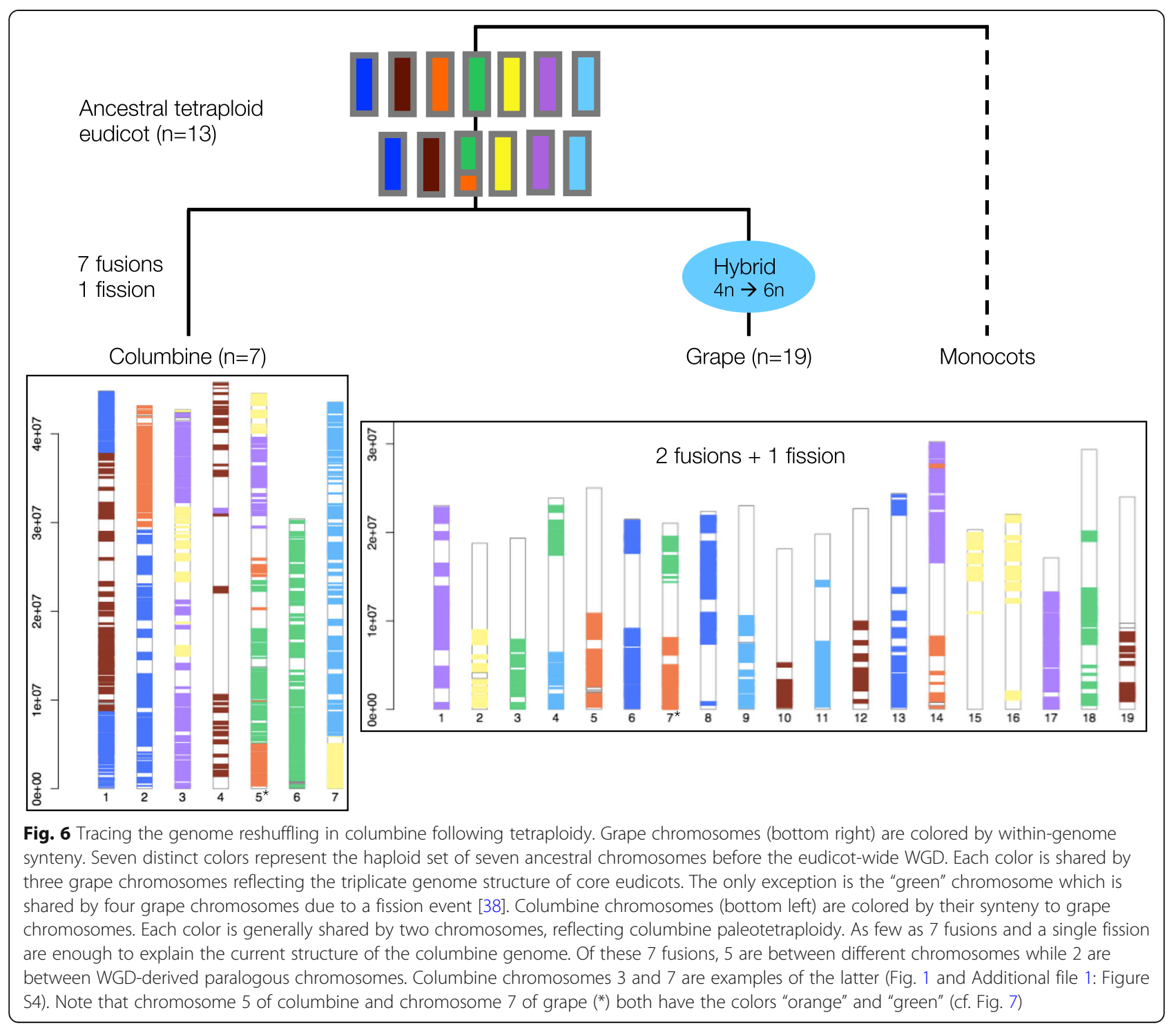




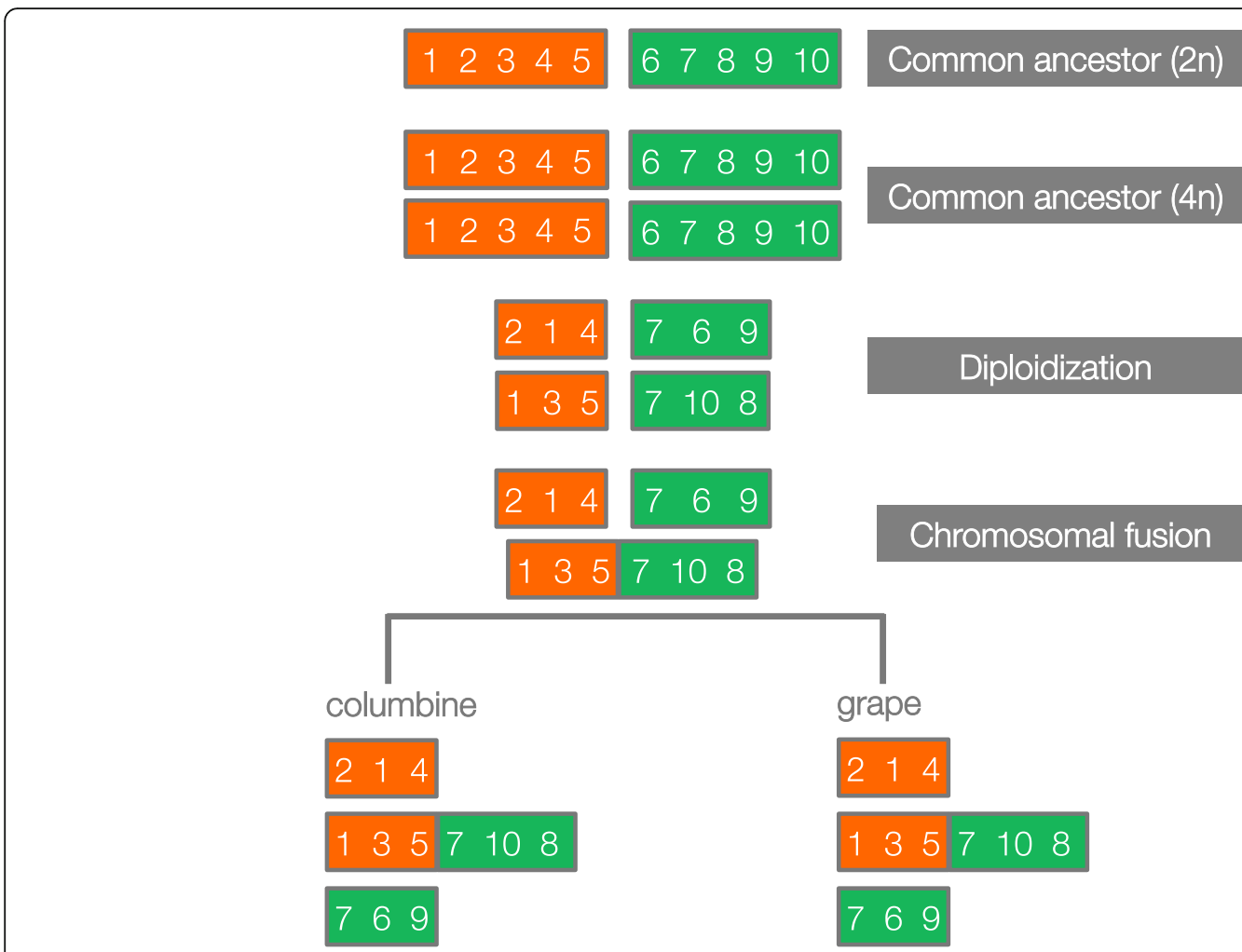

Fig. 7 Schematic of predicted synteny patterns in the case of shared ancestral fusion. Two ancestral chromosomes (orange and green rectangles, with genes depicted as numbers) undergo WGD. Paralogous chromosome pairs diverge as a part of the diploidization process. A fusion joins one version of the "orange" chromosome ("1,3,5") with one version of the "green" chromosome ("7, 10, 8.") If this took place in the common tetraploid ancestor of eudicots, the fused chromosomes in columbine and grape should also carry these versions on their "orange" and "green" portions. In the hypothetical example here, diploidization precedes the fusion event but may well happen afterwards with no effect on the predicted synteny patterns

clusters with that of cacao, strongly favoring a common origin of the fusion between "orange" and "green" ancestral chromosomes (Fig. 6).

\subsection{The core eudicots have a hybrid origin}

Our inference of shared tetraploidy between basal and core eudicots makes use of the signals presumably generated by diploidization (Figs. 5 and 7). However, hybridization of unreduced gametes from two divergent diploid genomes, "allotetraploidy," would also lead to gene order-based clustering between two different pairs of grape and columbine chromosomes (Additional file 1: Figure S11-S12). In this case, the alternative paralogous gene orders in the tetraploid ancestor reflect the gene orders on the progenitor chromosomes. Thus, the clustering pattern does not depend on whether the eudicot tetraploid genome evolved via "auto-" or "allopolyploidy." The same is not true for the second part of the process leading to hexaploidy. In this case, autopolyploidy would lead to the duplication of one of the existing chromosomes, whereas allohexaploidy would lead to one of the three paralogous grape chromosomes being an "outlier" with respect to the two grape-columbine pairing (Additional file 1: Figure S11-S12) - which is what we see in our data (Additional file 1: Figure S6-S8).

If our interpretation is correct and all core eudicots have a hybrid origin, the pattern of gene order-based clustering should be conserved. That is, we should be able to identify the hexaploidy-derived "outlier" chromosomes in other core eudicot genomes as well. To check this expectation, we again used the cacao genome, one of the most conserved genomes after grape $[9,39]$. Pairwise alignment between the homologous regions of columbine and cacao confirms our expectation: each member of columbine paralogs pairs up with a single cacao chromosome, leaving one of the cacao paralogs as an outlier (Additional file 1: Figure S13-S14). Furthermore, as shown in Fig. 8 (see also Additional file 1: Figure S10), the cacao regions putatively derived from tetraploidy and hexaploidy, respectively, show a very clear one-to-one match to those in grape (detected in the grape-columbine comparison). As expected, the putatively orthologous pairs of cacao and grape regions show similar levels of synteny conservation with their paralogous counterparts, with the "outlier" regions being the most divergent 


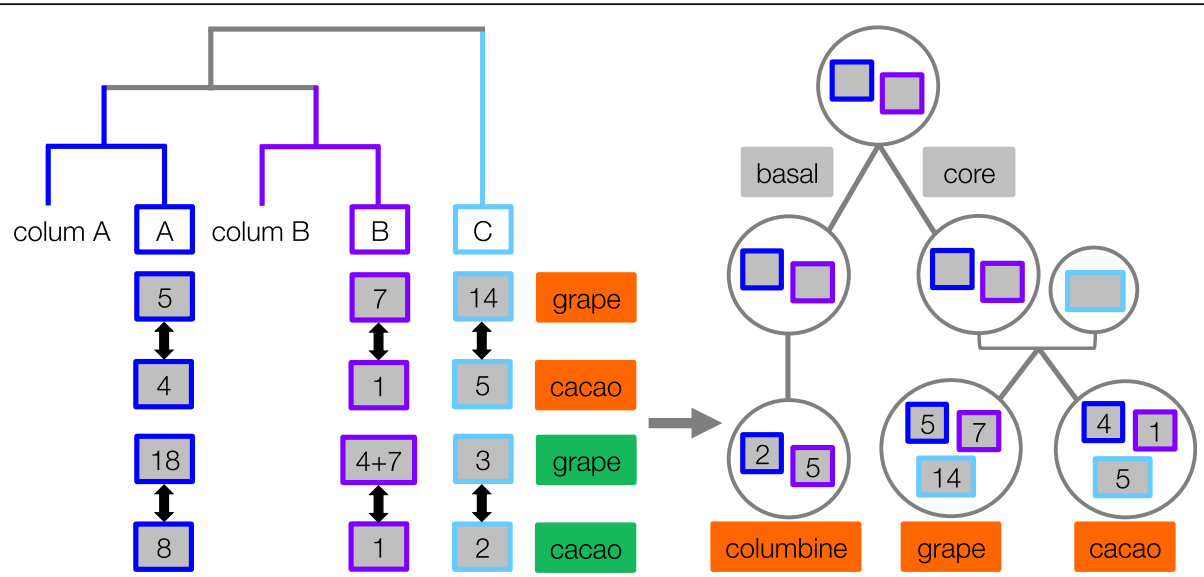

Fig. 8 The shared history of chromosomes in columbine, grape, and cacao. Gene order-based clustering results (left panel) are summarized here for the chromosomes harboring the "orange" and "green" homologous portions. The former corresponds to 5, 7, 14 in grape and 1, 4, 5 in cacao. The latter corresponds to 3, 4+ 7 (products of a fission), 18 in grape, and 1, 2, 8 in cacao. In columbine, the "orange" portions are on chromosomes 2 and 5 while the "green" portions are on chromosomes 6 and 5, each pair of which being denoted as colum A and colum B, respectively. Both grape- and cacao-columbine pairing distinguish tetraploidy-derived regions (blue and purple rectangles) from hybridizationderived ones (light blue rectangles), defining the orthologous sets of regions across the three eudicot genomes (right panel). The conservation of gene order exclusively between the putatively orthologous regions of grape and cacao (black arrows, Additional file 1: Figure S10) further strengthens our columbine-based inference of orthology

[38]. Thus, although not an independent source of evidence, the cacao genome supports a hybrid origin and highlights the key role of the columbine genome in unraveling the history of the eudicot genome.

\subsection{Current columbine chromosomes have mostly been generated via fusions}

It is widely accepted that genome shuffling post-WGD has shaped the present-day karyotypes of all plant genomes [34]. Nevertheless, the extent of genome shuffling as a part of the "re-diploidization" process seems to vary widely: only 3 chromosomal rearrangements post-gamma are enough to explain the current structure of the grape genome (Fig. 6) while almost 150 chromosomal rearrangements were necessary for the sunflower genome to reach its current karyotype after several rounds of WGD [11]. To check where columbine falls in this spectrum, we identified chromosomal rearrangements likely to have happened after the tetraploidy shared by all eudicots: if the pre-WGD ancestral eudicot karyotype had a haploid number of 7 chromosomes [28], only seven columbine-specific fusions and a single fission are enough to explain the reduction in columbine chromosome number from $n=13$ to $n=7$ after the ancestral fusion event (Fig. 6). These rearrangements involve all the chromosomes in columbine apart from chromosomes 4 and 6, the former of which paradoxically shows the greatest erosion of synteny with grape chromosomes (Fig. 6 and Additional file 1: Figure S3). Given all the evidence suggesting a "decaying" nature of columbine chromosome 4 [19], we repeated the analysis of grape-columbine synteny detection with relaxed parameter settings. We did this by decreasing the minimum number of aligned gene pairs within a block (from 5 to 3 ) and increasing the maximum genic distance between matches (from 20 to 30). This allowed us to extend the synteny blocks towards more proximal regions (Additional file 1: Figure S15). Further zooming into the synteny relationship between grape chromosomes that are homologous to columbine chromosome 4 confirmed that there is no evidence of a fusion event (Fig. 9).

The lack of a fusion event on columbine chromosome 6 might explain the fact that it is the smallest chromosome of columbine (Fig. 6). However, chromosome 4 is comparable in size to the remaining chromosomes, all of which are products of ancient fusion events. The observations that chromosome 4 has a higher proportion of genes in tandem duplicates ( 0.37 versus genome-wide mean of 0.22) and a greater extent of intra-chromosomal synteny (indicative of segmental duplications) (Additional file 1: Figure S17) suggest that chromosome 4 has reached a comparable size partly due to numerous tandem and segmental duplications and partly due to an expansion of repetitive DNA [19]. These results reinforce the idea that chromosome 4 has followed a distinct evolutionary path from the rest of the genome [19].

Fusion-dominated genome shuffling [34] is not the only facet of diploidization [40]. Following WGDs, gene duplicates get lost and this happens in a non-random manner. Genes involved in connected molecular functions like kinases, transcription factors, and ribosomal 


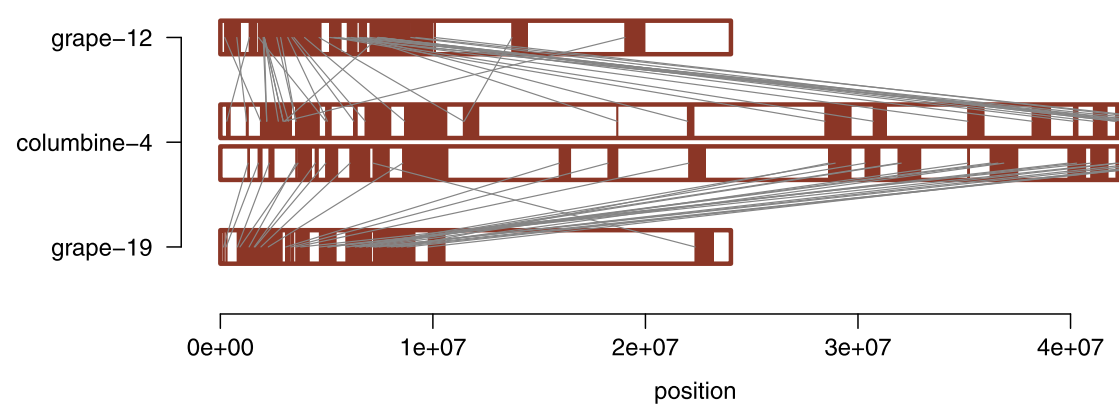

Fig. 9 Synteny between columbine chromosome 4 and grape chromosomes 12 and 19. Much smaller grape chromosomes look like the compact versions of columbine chromosome 4. Note that this result is generated with the most relaxed parameter combination in Additional file 1: Figure S15, but holds true for a less relaxed combination of parameters as well (Additional file 1: Figure S16)

proteins are retained in pairs [41-45] potentially due to dosage-related constraints [46]: losing or duplicating some, but not all of these dosage-sensitive genes, might upset the stoichiometric relationship between their protein products [47-49]. Consistent with this dosage balance hypothesis, columbine genes potentially retained post WGD (1302 genes across 76 syntenic regions; Additional file 2: Table S1) are enriched for the GO categories "structural constituent of ribosome," "transcription factor activity," "translation" $(p<0.001)$, and "protein tyrosine kinase activity" $(p<0.01)$. Tandemly duplicated genes $(n=6972)$, on the other hand, are depleted for the GO categories "structural constituent of ribosome" and "translation" $\left(p=10^{-17}\right)$, reflecting the role of dosagerelated purifying selection.

\section{Discussion}

All flowering plants are descended from a polyploid ancestor, and with only a few exceptions (e.g., Amborella from basal angiosperms [50]), all of them experienced at least one further round of WGD. Within-genome synteny (Fig. 1) shows that the columbine is an example of the latter, confirming the conclusions from other studies $[23,24,51]$. Here we show that this columbine tetraploidy is a remnant of a WGD at the base of all eudicots and is thus far more ancient than previously thought $[23,51]$. Furthermore, we use this observation to argue that the hexaploidy shared by the core eudicots must have involved allopolyploidy, i.e., presumably hybridization between the ancestral tetraploid and a diploid species.

A eudicot-wide WGD has been suggested by several studies [26, 35, 36, 52]. Our synteny-based approach solidifies these findings by demonstrating that the columbine and grape genomes have inherited the genome structure of a common tetraploid ancestor. That we can trace such an ancient tetraploidy is due to two facts. First, the genome structure of columbine is wellpreserved and free from recent WGDs. Second, genomes provide much greater information than genes alone.
Indeed, a recent study on another basal eudicot [51], the opium poppy, highlights these two facts. Having experienced a recent WGD ( $\sim 8$ million years ago), the genome of the poppy is dominated by syntenic gene pairs of low divergence (Fig. 1C in [51]), although it also carries highly diverged paralogs whose Ks values nicely overlap with our estimates for columbine and grape, consistent with an eudicot-wide WGD (compare Fig. S13D in [51] to Fig. 4). However, the strength of the signal from recent polyploidization largely obscures the much weaker signal of ancient polyploidization (interpreted as segmental duplications by Guo et al. [51]). In fact, although overlooked by the authors, the intergenomic synteny between columbine and poppy provides a clear signature of an eudicot-wide WGD (Fig. S9D in [51]). Differing from columbines with only one additional genome duplication, the poppy genome aligns to the columbine genome in a 4:2 manner, with 4 paralogous regions of poppy syntenic to 2 paralogous regions of columbine derived from the ancient shared tetraploidy.

Our approach also helps us shed light on the nature of the gamma hexaploidy found in all core eudicots ([9, 28-32], and Supplementary Note 5 in [33]). WGDs have often been discussed as if they were "events," ignoring the process by which they originated. We show here that core eudicot hexaploidy is the result of two processes: an ancient tetraploidization shared by all eudicots, followed by allopolyploidization leading to the core eudicots. In other words, all core eudicots have a hybrid origin. An allohexaploid origin has indeed been previously suggested by Murat et al. [9], who identified the three subgenomes of grape using differential patterns of gene loss on "dominant" versus "sensitive" subgenomes. Their classification assumes that the most recently added set of paralogous chromosomes will be "dominant," because they have spent a shorter amount of time in the polyploid genome and thus experienced fewer gene losses. Contrary to this, our results suggest that the most recently added grape chromosomes (chromosomes 3, 8, 9, and 14) largely correspond to the "sensitive" grape chromosomes identified by Murat et al. [9]. Instead, we argue that the extensive gene loss in the most 
recently added subgenome reflects its divergence from the other two subgenomes at the time of hexaploid formation, perhaps similar to the situation in the allotetraploid Arabidopsis suecica, which is a hybrid between the more ancestrallike $(n=8)$ genome of $A$. arenosa and the heavily reduced $(n=5)$ genome of $A$. thaliana [53]. Another example is hexaploid wheat, which is a hybrid between tetraploid emmer wheat and wild diploid grass, Aegilops tauschii ([54] and references therein]).

\section{Conclusions}

Our findings reveal the hybrid structure of core eudicot genomes and will hopefully help us understand what hybridization has meant for core eudicots-a group which comprises more than $70 \%$ of all living flowering plants [55]. What are the hybridization-coupled changes that have led to the current patterns of gene expression, methylation, or transposable element density/distribution? All these questions call for additional genomes from basal eudicots which-as this study illustrateshave great values as outgroup to the core eudicots. More data will also allow the development of sophisticated analysis methods based on explicit models of the evolution of gene order, which our results suggest is a very powerful source of information about the past.

\section{Materials and methods}

\subsection{Synteny detection}

We performed all genes (CDS)-against-all genes (CDS) BLAST for the latest version of Aquilegia coerulea reference genome (v3.1) using the SynMap tool [29] in the online CoGe portal [56]. We also looked at the synteny within Vitis vinifera (v12) and between A. coerulea and $V$. vinifera using default parameter combinations in DAGChainer. We filtered the raw output files for both within-grape and columbine-to-grape synteny. For the former, we only kept the blocks that are syntenic between the polyploidy-derived paralogous chromosomes of grape as identified by Jaillon et al. [28] (Additional file 4: Table S3). For the latter, we required that a given columbine chromosome is overall syntenic to all the three paralogous chromosomes of grape (Additional file 3: Table S2). So, for a given pair of columbine and grape chromosomes, we only kept the blocks if the columbine chromosome also matches to the other members of paralogous grape chromosomes.

The raw output files can be regenerated at the CoGe portal [56] using the id numbers provided below for each species (Availability of data and materials) and changing the default parameter combination in DAGChainer ( $D: A=20: 5)$ when needed. $D$ and A specify the maximum genic distance between two matches and the minimum number of aligned gene pairs, respectively, to form a collinear syntenic block.

\subsection{Estimating the divergence between synteny block pairs}

We used Ks (the number of synonymous substitutions per synonymous site) values provided for each homolog gene pair by the CoGe portal [56]. We estimated the median Ks of homologous genes in a synteny block after filtering gene pairs with $\mathrm{Ks}>10$ due to a saturation effect [57]. Both values are provided in Supplementary Data 1-3 for within columbine, columbine-to-grape, and within-grape synteny, respectively.

\subsection{Quantifying gene order similarity}

We "reconstructed" a given set of columbine and grape chromosomes at their homologous regions (color-coded in Fig. 6). We seeded this reconstruction by focusing on at least three consecutive genes aligning between a pair of columbine and grape chromosomes ( $\mathrm{D}: \mathrm{A}=0: 3$ ). We particularly chose three genes since it is the most stringent value we could use to detect homologous synteny blocks; we detected almost nothing when we required 4 consecutive genes $(D: A=0: 4)$. This stringent criteria aim to minimize the effect of gene movement on the homology between columbine and grape chromosomes. Once we had the list of genes, we then looked for their paralogous counterparts on the remaining columbine and grape chromosomes using intragenomic gene-togene blast $(\mathrm{D}: \mathrm{A}=0: 1)$. Having chromosomes represented by syntenic gene sets and reminiscent of these sets (Additional file 1: Figure S5 and Additional file 5: Table S4), we assigned a unique word to each synteny block and the genes forming the block to be able to use the text alignment provided by the $\mathrm{R}$ package align_local [58]. We then quantified the gene ("word") similarity as such: for an initial $\mathrm{N}$ number of words on a columbine chromosome ( $\mathrm{N}$ = window size), we did a pairwise alignment between these $\mathrm{N}$ words and all the words a grape chromosome (match $=4$, gap $=-1)$. We repeated the same analysis with the inverted order of $\mathrm{N}$ words and picked the maximum alignment score. We repeated these steps by sliding the window by one word and keeping the $\mathrm{N}$ constant to get a distribution of scores as in Additional file 1: Figure S6-S8. We used different N values ranging from 4 to 15 . Note that we excluded columbine chromosomes 3, 4, and 7 from this analysis since they all have a complex history of lineage-specific chromosomal reshuffling events (Figs. 1, 6, Additional file 1: Figure S4 and S17).

We applied the same stringent criteria (D:A = 0:3) to detect the homologous regions between grape and cacao (Theobroma cacao, v1). The same criteria led to very few homologous regions between columbine and cacao. So, we relaxed the parameters for the synteny detection between these two genomes ( $D: A=0: 2)$ and quantified the gene order similarity with greater window sizes $(N=20$, 
$30,35,40$, and 50). Note that we focused on the triplicated regions distributed across 3 different cacao chromosomes (Fig. 8, Additional file 1: Figure S13-S14), which are rather unaffected by lineage-specific shuffling [38].

\subsection{Statistical testing of gene order similarity}

Given the gene order similarity between the two different pairs of columbine and grape chromosomes harboring homologous regions, we performed permutation tests to estimate the probability of observing such a clustering just by chance. To do so, we first combined all the grape genes and sampled the same number of genes ("words") as we observe to reconstruct each of the paralogous grape chromosome. We repeated the quantification step as above to get a permuted distribution of alignment score between a pair of columbine and grape chromosomes. We used the Wilcoxon rank sum test (W-statistic) to quantify the shift in the distribution of alignment scores between one of the members of columbine paralogous chromosomes and its best grape hit when combined with the alignment scores between the same columbine chromosome and other grape chromosomes. We repeated the same analysis for the other member of columbine paralogous chromosomes as well. Having these observed Wstatistics, we counted the number of cases (out of 100) where the permuted distributions generate $\mathrm{W}$-statistics as high as or higher than the observed ones. We ran permutation tests for the columbine-cacao pairing as well (Additional file 1: Figure S13-S14).

\subsection{Building gene trees}

We built upgma trees for the homologous genes (Additional file 6: Table S5) distributed across a given set of columbine and grape chromosomes (color-coded in Fig. 6). We first detected homologous genes aligning between a pair of columbine and grape chromosomes ( $D: A=0: 1$ ). We then searched for their paralogous counterparts using intragenomic blasts ( $\mathrm{D}: \mathrm{A}=0: 1)$. For protein alignment, we required at least five homologous genes, each from a single chromosome in the given set and ran ClustalW2 (v2.1) with the options -TREE -KIMURA -CLUSTERING = UPGMA -OUTPUTTREE = dist [59]. Of all the trees generated by ClustalW2 (informative trees), we only focused on the ones that support the synteny-based pairings (Additional file 1: Figure S6-S8), which are detected by the subtrees function in $\mathrm{R}$ package ape $[60,61]$. Once we had the sets of homologous genes from this subset of trees, we assigned a unique word to the each set and quantified the gene order similarity between pairs of columbine and grape chromosomes as mentioned above.

For protein sequences, we used the annotations provided by JGI and Ensembl for columbine [19] and grape [62], respectively. Note that CoGe [56] outputs grape genes with the "PAC" tag while they are tagged with
Table $12 \times 2$ contingency table obtained by classifying genes into 2 categorical variables. The letters denote the number of genes for a given category (e.g., "a" denotes the number of retained genes annotated with the tested $\mathrm{GO}$ category)

\begin{tabular}{llll}
\hline & GO & Not-GO & SUM \\
\hline Retained & $a$ & $b$ & $a+b^{*}$ \\
Not-retained & $c$ & $d$ & $c+d$ \\
Sum & $a+c$ & $b+d$ & $N=$ total number of genes \\
& & & $=29,550$ (across 7 chromosomes)
\end{tabular}

*equal to 1302 and 6972 for candidate WGD-derived paralogs and tandem gene duplicates, respectively

"VIT" in the Ensembl database. To match these different ids, we used two intermediary files. The first one is a gff file provided by CoGe (available at https://genomevolution.org/coge/GenomeInfo.pl?gid=19990). The second one is a conversion file provided by the Grape Genome Database [63] that lists the correspondence between different gene ids (can be downloaded from http://genomes.cribi.unipd.it/DATA/). These two files contain the common tag "GSVIVT" which bridges the "PAC" and "VIT" tags.

\subsection{GO enrichment analysis}

We used gene annotations provided by JGI [19] to test the null hypothesis that the property for a gene to be retained post-WGD and to belong to a given GO category are independent. We created a $2 \times 2$ contingency table (Table 1) and applied Fisher's exact test for each GO category independently. We repeated the same analysis for tandem gene duplicates as identified by SynMap $[29,56]$; this time, testing the null hypotheses that the property for a gene to be tandemly duplicated and to belong to a given GO category are independent. We excluded genes on scaffolds and reported enriched/depleted categories if they remain significant $(p<0.05)$ after multiple test correction $(\mathrm{fdr})$.

\section{Supplementary information}

Supplementary information accompanies this paper at https://doi.org/10. 1186/s13059-019-1888-8.

\section{Additional file 1: Supplementary Figure S1-S17.}

Additional file 2: Table S1. Synteny blocks in the columbine genome. Additional file 3: Table S2. Synteny blocks between columbine and grape chromosomes.

Additional file 4: Table S3. Synteny blocks in the grape genome. Additional file 5: Table S4. Reconstruction of columbine and grape chromosomes.

Additional file 6: Table S5. Homologous genes between columbine and grape.

Acknowledgements

We thank Robin Burns and Claus Vogl for their comments on the manuscript; Daniel Gómez Sánchez and Benjamin Jaegle for the fruitful discussions. 


\section{Peer review information}

Andrew Cosgrove and Barbara Cheifet were the primary editors on this article and managed its editorial process and peer review in collaboration with the rest of the editorial team.

\section{Authors' contributions}

GA performed all analyses. GA and MN wrote the manuscript. Both authors read and approved the final manuscript.

\section{Funding}

G.A. was supported by the Vienna Graduate School of Population Genetics (Austrian Science Fund, FWF: DK W1225-B20)

\section{Availability of data and materials}

The columbine, grape, and cacao genomes are available at the CoGE porta for the synteny analyses with the id numbers 28620, 19990, and 25287, respectively [56]

\section{Ethics approval and consent to participate} Not applicable.

\section{Competing interests}

The authors declare no competing interests.

\section{Author details}

${ }^{1}$ Gregor Mendel Institute, Austrian Academy of Sciences, Vienna Biocenter, Vienna, Austria. ${ }^{2}$ Vienna Graduate School of Population Genetics, Vienna, Austria.

Received: 11 July 2019 Accepted: 14 November 2019

Published online: 28 November 2019

\section{References}

1. Wendel JF. The wondrous cycles of polyploidy in plants. Am J Bot. 2015;102: 1753-6.

2. Van de Peer Y, Mizrachi E, Marchal K. The evolutionary significance of polyploidy. Nat Rev Genet. 2017;18:411-24.

3. Jiao Y, Wickett NJ, Ayyampalayam S, Chanderbali AS, Landherr L, Ralph PE, et al. Ancestral polyploidy in seed plants and angiosperms. Nature. 2011; 473:97-100

4. Leitch IJ, Bennett MD. Genome downsizing in polyploid plants. Biol J Linn Soc Lond. Oxford University Press. 2004;82:651-63.

5. Comai L. The advantages and disadvantages of being polyploid. Nat Rev Genet. 2005:6:836-46.

6. Thomas BC, Pedersen B, Freeling M. Following tetraploidy in an Arabidopsis ancestor, genes were removed preferentially from one homeolog leaving clusters enriched in dose-sensitive genes. Genome Res. 2006;16:934-46.

7. Otto SP. The evolutionary consequences of polyploidy. Cell. 2007:131:45262.

8. Renny-Byfield S, Chester M, Kovařík A, Le Comber SC, Grandbastien M-A, Deloger $\mathrm{M}$, et al. Next generation sequencing reveals genome downsizing in allotetraploid Nicotiana tabacum, predominantly through the elimination of paternally derived repetitive DNAs. Mol Biol Evol. 2011;28:2843-54.

9. Murat F, Zhang R, Guizard S, Gavranović H, Flores R, Steinbach D, et al. Karyotype and gene order evolution from reconstructed extinct ancestors highlight contrasts in genome plasticity of modern rosid crops. Genome Biol Evol. 2015;7:735-49.

10. Murat F, Armero A, Pont C, Klopp C, Salse J. Reconstructing the genome of the most recent common ancestor of flowering plants. Nat Genet. 2017:49: 490-6.

11. Badouin H, Gouzy J, Grassa CJ, Murat F, Staton SE, Cottret L, et al. The sunflower genome provides insights into oil metabolism, flowering and Asterid evolution. Nature. 2017;546:148-52.

12. Blanc $\mathrm{G}$, Wolfe $\mathrm{KH}$. Functional divergence of duplicated genes formed by polyploidy during Arabidopsis evolution. Plant Cell. 2004;16:1679-91.

13. De Smet R, Adams KL, Vandepoele K, Van Montagu MCE, Maere S, Van de Peer $Y$. Convergent gene loss following gene and genome duplications creates single-copy families in flowering plants. Proc Natl Acad Sci U S A. 2013;110:2898-903.

14. Woodhouse MR, Schnable JC, Pedersen BS, Lyons E, Lisch D, Subramaniam S, et al. Following Tetraploidy in Maize, a Short Deletion Mechanism
Removed Genes Preferentially from One of the Two Homeologs. PLoS Biol. Public Library of Science; 2010;8:e1000409.

15. Schnable JC, Springer NM, Freeling M. Differentiation of the maize subgenomes by genome dominance and both ancient and ongoing gene loss. Proc Natl Acad Sci U S A. 2011;108:4069-74.

16. Tang H, Woodhouse MR, Cheng F, Schnable JC, Pedersen BS, Conant G, et al. Altered patterns of fractionation and exon deletions in Brassica rapa support a two-step model of paleohexaploidy. Genetics. 2012;190:1563-74.

17. Hodges SA, Kramer EM. Columbines. Curr Biol. 2007;17:R992-4.

18. Soltis DE, Smith SA, Cellinese N, Wurdack K, Tank DC, Brockington SF, et al. Angiosperm phylogeny: 17 genes, 640 taxa. Am J Bot. 2011;98:704-30.

19. Filiault DL, Ballerini ES, Mandáková T, Aköz G, Derieg NJ, Schmutz J, et al. The Aquilegia genome provides insight into adaptive radiation and reveals an extraordinarily polymorphic chromosome with a unique history. Elife. 2018;7. https://doi.org/10.7554/eLife.36426.

20. Lynch $\mathrm{M}$, Conery JS. The evolutionary fate and consequences of duplicate genes. Science. 2000;290:1151-5.

21. Blanc $G$, Wolfe KH. Widespread paleopolyploidy in model plant species inferred from age distributions of duplicate genes. Plant Cell. 2004;16:1667-78.

22. Cui L, Wall PK, Leebens-Mack JH, Lindsay BG, Soltis DE, Doyle JJ, et al. Widespread genome duplications throughout the history of flowering plants. Genome Res. 2006;16:738-49.

23. Vanneste $K$, Baele G, Maere S, Van de Peer Y. Analysis of 41 plant genomes supports a wave of successful genome duplications in association with the Cretaceous-Paleogene boundary. Genome Res. 2014:24:1334-47.

24. Tiley GP, Ané C, Burleigh JG. Evaluating and Characterizing Ancient WholeGenome Duplications in Plants with Gene Count Data. Genome Biol Evol. 2016:8:1023-37.

25. Doyle JJ, Egan AN. Dating the origins of polyploidy events. New Phytol. 2010;186:73-85.

26. Jiao Y, Paterson AH. Polyploidy-associated genome modifications during land plant evolution. Philos Trans R Soc Lond B Biol Sci. 2014;369. https:// doi.org/10.1098/rstb.2013.0355

27. Melters DP, Bradnam KR, Young HA, Telis N, May MR, Ruby JG, et al. Comparative analysis of tandem repeats from hundreds of species reveals unique insights into centromere evolution. Genome Biol. 2013;14:R10.

28. Jaillon O, Aury J-M, Noel B, Policriti A, Clepet C, Casagrande A, et al. The grapevine genome sequence suggests ancestral hexaploidization in major angiosperm phyla. Nature. 2007:449:463-7.

29. Lyons E, Pedersen B, Kane J, Freeling M. The Value of Nonmodel Genomes and an Example Using SynMap Within CoGe to Dissect the Hexaploidy that Predates the Rosids. Trop Plant Biol. 2008:1:181-90.

30. Potato Genome Sequencing Consortium, Xu X, Pan S, Cheng S, Zhang B, Mu D, et al. Genome sequence and analysis of the tuber crop potato. Nature. 2011:475:189-95.

31. Truco MJ, Ashrafi H, Kozik A, van Leeuwen H, Bowers J, Wo SRC, et al. An Ultra-High-Density, Transcript-Based, Genetic Map of Lettuce. G3. 2013;3: 617-31

32. Denoeud F, Carretero-Paulet L, Dereeper A, Droc G, Guyot R, Pietrella M, et al. The coffee genome provides insight into the convergent evolution of caffeine biosynthesis. Science. 2014;345:1181-4.

33. Bombarely A, Moser M, Amrad A, Bao M, Bapaume L, Barry CS, et al. Insight into the evolution of the Solanaceae from the parental genomes of Petunia hybrida. Nat Plants. 2016;2:16074.

34. Salse J. Ancestors of modern plant crops. Curr Opin Plant Biol. 2016:30:134-42

35. Ming R, VanBuren R, Liu Y, Yang M, Han Y, Li L-T, et al. Genome of the longliving sacred lotus (Nelumbo nucifera Gaertn.). Genome Biol. 2013;14:R41.

36. Jiao Y, Leebens-Mack J, Ayyampalayam S, Bowers JE, McKain MR, McNeal J, et al. A genome triplication associated with early diversification of the core eudicots. Genome Biol. 2012:13:R3.

37. Zeng L, Zhang Q, Sun R, Kong H, Zhang N, Ma H. Resolution of deep angiosperm phylogeny using conserved nuclear genes and estimates of early divergence times. Nat Commun. 2014;5:4956.

38. Zheng C, Chen E, Albert VA, Lyons E, Sankoff D. Ancient eudicot hexaploidy meets ancestral eurosid gene order. BMC Genomics. 2013;14(Suppl 7):S3.

39. Argout X, Salse J, Aury J-M, Guiltinan MJ, Droc G, Gouzy J, et al. The genome of Theobroma cacao. Nat Genet. 2011:43:101-8.

40. Soltis DE, Visger CJ, Marchant DB, Soltis PS. Polyploidy: Pitfalls and paths to a paradigm. Am J Bot. 2016;103:1146-66.

41. Seoighe C, Wolfe $\mathrm{KH}$. Yeast genome evolution in the post-genome era. Curr Opin Microbiol. 1999;2:548-54. 
42. Tian C-G, Xiong Y-Q, Liu T-Y, Sun S-H, Chen L-B, Chen M-S. Evidence for an ancient whole-genome duplication event in rice and other cereals. Yi Chuan Xue Bao. 2005;32:519-27.

43. Aury J-M, Jaillon O, Duret L, Noel B, Jubin C, Porcel BM, et al. Global trends of whole-genome duplications revealed by the ciliate Paramecium tetraurelia. Nature. 2006:444:171-8.

44. Schnable PS, Ware D, Fulton RS, Stein JC, Wei F, Pasternak S, et al. The B73 maize genome: complexity, diversity, and dynamics. Science. 2009;326:1112-5.

45. Rodgers-Melnick E, Mane SP, Dharmawardhana P, Slavov GT, Crasta OR, Strauss SH, et al. Contrasting patterns of evolution following whole genome versus tandem duplication events in Populus. Genome Res. 2012;22:95-105.

46. Birchler JA, Bhadra U, Bhadra MP, Auger DL. Dosage-dependent gene regulation in multicellular eukaryotes: implications for dosage compensation, aneuploid syndromes, and quantitative traits. Dev Biol. 2001; 234:275-88.

47. Papp B, Pál C, Hurst LD. Dosage sensitivity and the evolution of gene families in yeast. Nature. 2003:424:194-7.

48. Freeling M, Thomas BC. Gene-balanced duplications, like tetraploidy, provide predictable drive to increase morphological complexity. Genome Res. 2006;16:805-14.

49. Birchler JA, Veitia RA. Gene balance hypothesis: connecting issues of dosage sensitivity across biological disciplines. Proc Natl Acad Sci U S A. 2012;109: 14746-53.

50. Amborella Genome Project. The Amborella genome and the evolution of flowering plants. Science. 2013;342:1241089.

51. Guo L, Winzer T, Yang X, Li Y, Ning Z, He Z, et al. The opium poppy genome and morphinan production. Science. 2018. https://doi.org/10.1126/ science.aat4096

52. Malacarne G, Perazzolli M, Cestaro A, Sterck L, Fontana P, Van de Peer Y, et al. Deconstruction of the (paleo)polyploid grapevine genome based on the analysis of transposition events involving NBS resistance genes. PLoS One. 2012;7:e29762.

53. O'Kane SL, Schaal BA, Al-Shehbaz IA. The Origins of Arabidopsis suecica (Brassicaceae) as Indicated by Nuclear rDNA Sequences. Syst Bot. 1996;21:559-66.

54. Matsuoka Y. Evolution of polyploid triticum wheats under cultivation: the role of domestication, natural hybridization and allopolyploid speciation in their diversification. Plant Cell Physiol. 2011;52:750-64.

55. Friis EM, Pedersen KR, Crane PR. The emergence of core eudicots: new floral evidence from the earliest Late Cretaceous. Proc Biol Sci. 2016;283. https:// doi.org/10.1098/rspb.2016.1325.

56. Lyons E, Freeling M. How to usefully compare homologous plant genes and chromosomes as DNA sequences. Plant J. 2008:53:661-73.

57. Blanc $G$, Hokamp K, Wolfe KH. A recent polyploidy superimposed on older largescale duplications in the Arabidopsis genome. Genome Res. 2003;13:137-44.

58. Smith DA, Cordell R, Dillon EM. Infectious texts: Modeling text reuse in nineteenth-century newspapers. 2013 IEEE International Conference on Big Data; 2013. p. 86-94.

59. Larkin MA, Blackshields G, Brown NP, Chenna R, McGettigan PA, McWilliam $H$, et al. Clustal W and Clustal X version 2.0. Bioinformatics. 2007;23:2947-8.

60. R Core Team. R: A language and environment for statistical computing. Vienna: R Foundation for Statistical Computing; 2013. Available from: https://www.R-project.org/

61. Paradis E, Claude J, Strimmer K. APE: Analyses of Phylogenetics and Evolution in R language. Bioinformatics. 2004;20:289-90.

62. Kersey PJ, Allen JE, Allot A, Barba M, Boddu S, Bolt BJ, et al. Ensembl Genomes 2018: an integrated omics infrastructure for non-vertebrate species. Nucleic Acids Res. 2018:46:D802-8.

63. Vitulo N, Forcato C, Carpinelli EC, Telatin A, Campagna D, D'Angelo M, et al. A deep survey of alternative splicing in grape reveals changes in the splicing machinery related to tissue, stress condition and genotype. BMC Plant Biol. 2014;14:99.

\section{Publisher's Note}

Springer Nature remains neutral with regard to jurisdictional claims in published maps and institutional affiliations.

\section{Ready to submit your research? Choose BMC and benefit from:}

- fast, convenient online submission

- thorough peer review by experienced researchers in your field

- rapid publication on acceptance

- support for research data, including large and complex data types

- gold Open Access which fosters wider collaboration and increased citations

- maximum visibility for your research: over $100 \mathrm{M}$ website views per year

At BMC, research is always in progress.

Learn more biomedcentral.com/submissions 